\title{
Microscopic Processes in Global Relativistic Jets Containing Helical Magnetic Fields
}

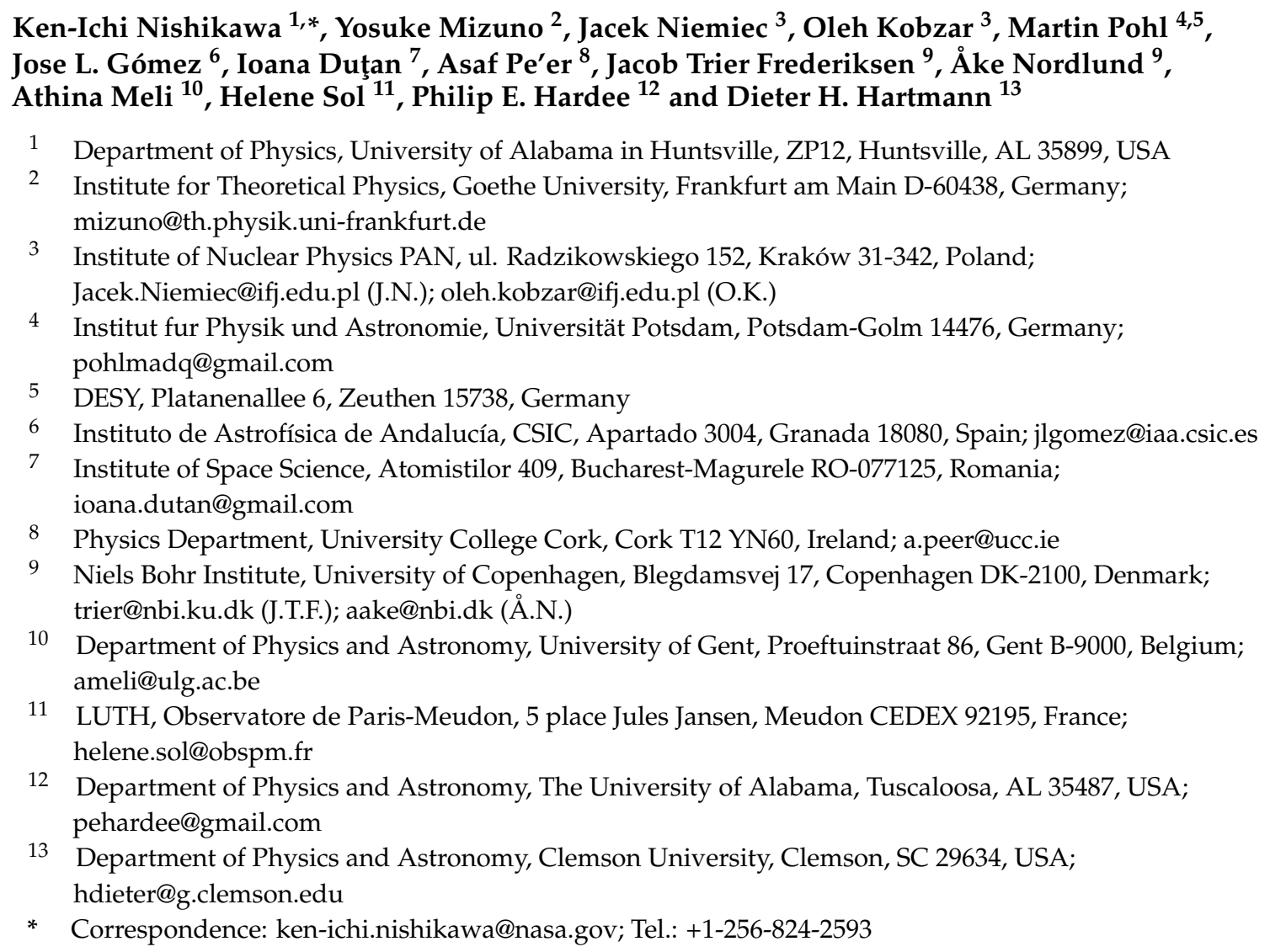

Academic Editors: Jose L. Gòmez, Alan P. Marscher and Svetlana G. Jorstad

Received: 13 July 2016; Accepted: 22 September 2016; Published: 29 September 2016

\begin{abstract}
In the study of relativistic jets one of the key open questions is their interaction with the environment on the microscopic level. Here, we study the initial evolution of both electron-proton $\left(e^{-}-p^{+}\right)$and electron-positron $\left(e^{ \pm}\right)$relativistic jets containing helical magnetic fields, focusing on their interaction with an ambient plasma. We have performed simulations of "global" jets containing helical magnetic fields in order to examine how helical magnetic fields affect kinetic instabilities such as the Weibel instability, the kinetic Kelvin-Helmholtz instability (kKHI) and the Mushroom instability (MI). In our initial simulation study these kinetic instabilities are suppressed and new types of instabilities can grow. In the $e^{-}-p^{+}$jet simulation a recollimation-like instability occurs and jet electrons are strongly perturbed. In the $e^{ \pm}$jet simulation a recollimation-like instability occurs at early times followed by a kinetic instability and the general structure is similar to a simulation without helical magnetic field. Simulations using much larger systems are required in order to thoroughly follow the evolution of global jets containing helical magnetic fields.
\end{abstract}

Keywords: relativistic jets; particle-in-cell simulations; global jets; helical magnetic fields; kinetic instabilities; kink instability 


\section{Introduction}

Relativistic jets are collimated plasma outflows associated with active galactic nuclei (AGNs), gamma-ray bursts (GRBs), and pulsars. Among these astrophysical systems, blazars and GRB jets produce the most luminous phenomena in the universe (e.g., [1] ). Despite extensive observational and theoretical investigations (including simulation studies), our understanding of their formation, interaction, and evolution in the ambient plasma-and consequently their observable properties, such as time-dependent flux and polarity-remains quite limited. One of the key open questions in the study of relativistic jets is how they interact with the immediate plasma environment on the microscopic scale. In particular, we wish to examine how relativistic jets containing helical magnetic fields evolve under the influence of kinetic and MHD-like instabilities that occur within and at the jet boundaries, with consequences such as flares due to reconnection.

Jet outflows are commonly thought to be dynamically hot (relativistic) magnetized plasma flows launched, accelerated, and collimated in regions where Poynting flux dominates over particle (matter) flux [2,3]. This scenario involves a helical large-scale magnetic field structure in some AGN jets, which provides a unique signature in the form of observed asymmetries across the jet width, particularly in the polarization [4-6].

Large-scale ordered magnetic fields have been invoked to explain the launching, acceleration, and collimation of relativistic jets from the central nuclear region of an active galaxy [7], and from coalescing and merging compact objects (neutron stars and black holes); e.g., [8]. The magnetic field structure and particle composition of the jets are still not well constrained observationally. Circular polarization (CP; measured as Stokes parameter V) in the radio continuum emission from AGN jets provides a powerful diagnostic for the deduction of magnetic structure and particle composition, because - unlike linear polarization (LP) - CP is expected to remain almost completely unmodified by external screens (e.g., [9]).

Jet particle composition has remained an unresolved issue ever since the discovery of jets. The two main candidates are a "normal" plasma consisting of relativistic electrons and protons (an $e^{-}-p^{+}$jet), and a "pair" plasma consisting only of relativistic electrons and positrons (an $e^{ \pm}$jet) [10]. The detection of circular polarization from the violently variable quasar 3C 279 at several epochs, using the Very Long Baseline Array (VLBA) at $15 \mathrm{GHz}$, has been used by Wardle et al. [10] to argue that the circular polarization is produced by Faraday conversion of linear to circular polarization in the jet plasma. This conversion requires that the energy distribution of the radiating particles extends down to $\gamma_{\min } \ll 100$, and that should imply an $e^{ \pm}$jet.

Over the past few years, we have been using a fully self-consistent relativistic particle-in-cell (RPIC) simulation method to investigate collisionless shocks, and the kinetic Kelvin-Helmholtz instability $(\mathrm{kKHI})$ at relativistic jet-sheath shear boundaries, and to calculate the resulting synthetic emission spectra. The RPIC code used in these studies is a modified version of the TRISTAN code [11], parallelized with MPI and utilized for various research projects (e.g., [12-14]). To date, RPIC simulations of the kKHI have been performed in slab [15-24] and cylindrical geometries using periodic boundary conditions [25,26]. Previously, full-scale shock simulations have not incorporated velocity shear interactions at the jet boundary with the ambient plasma (interstellar medium) (e.g., [13]), and global shock simulations including velocity shear interactions performed to date used only very small simulation boxes [27-29]. Recently, we performed "global" jet simulations involving the injection of a cylindrical unmagnetized jet into an ambient plasma in order to investigate shock (Weibel instability) and velocity shear instabilities (kKHI and Mushroom instability (MI)) simultaneously [14]. Here we report preliminary results of our new studies of global relativistic jets containing helical magnetic fields.

\section{Global Jet Simulations}

Jets generated from black holes and injected into the ambient interstellar medium contain magnetic fields which are thought to be helical. Therefore, we perform global simulations of jets containing 
helical magnetic fields injected into an ambient medium (e.g., [3]). The key issue we investigate is how the helical magnetic fields affect the growth of the kKHI, the MI, and the Weibel instability. It is known from RMHD simulations that jets containing helical magnetic fields develop kink instability (e.g., [30,31]). Recently, it has been demonstrated by Markidis et al. [32] that a kinked jet structure leads to the occurrence of a secondary reconnection.

\subsection{Helical Magnetic Field Structure}

In our simulations, cylindrical jets are injected with a helical magnetic field (see Figure 1a) [32] implemented like that in RMHD simulations performed by Mizuno et al. [33]. Our toroidal magnetic field structure is similar to a simple screw-pinch configuration adopted by [32] with the radially dependent axial magnetic field. However, our simulations use Cartesian coordinates. Since $\alpha=1$, Equations (9)-(11) from [33] are reduced to Equation (1), and the magnetic field takes the form:

$$
B_{x}=\frac{B_{0}}{\left[1+(r / a)^{2}\right]}, \quad B_{\phi}=\frac{(r / a) B_{0}}{\left[1+(r / a)^{2}\right]}
$$

The toroidal magnetic field is created by a current $+J_{x}(y, z)$ in the positive $x$-direction, so that defined in Cartesian coordinates:

$$
B_{y}(y, z)=\frac{\left(\left(z-z_{\mathrm{jc}}\right) / a\right) B_{0}}{\left[1+(r / a)^{2}\right]}, \quad B_{z}(y, z)=-\frac{\left(\left(y-y_{\mathrm{jc}}\right) / a\right) B_{0}}{\left[1+(r / a)^{2}\right]} .
$$

Here $a$ is the characteristic length-scale of the helical magnetic field, $\left(y_{\mathrm{jc}}, z_{\mathrm{jc}}\right)$ is the center of the jet, and $r=\sqrt{\left(y-y_{\mathrm{jc}}\right)^{2}+\left(z-z_{\mathrm{jc}}\right)^{2}}$. The chosen helicity is defined through Equation (2), which is left-handed polarity with positive $B_{0}$. At the jet orifice, we implement the helical magnetic field without the motional electric fields. This corresponds to a toroidal magnetic field generated self-consistently by jet particles moving along the $+x$-direction.

\subsection{Currents and Fields in Helically Magnetized RPIC Jets}

As an initial step, we have examined how the helical magnetic field modifies jet evolution using a small system before performing larger-scale simulations, and a schematic of the simulation injection setup is shown in Figure 1a.
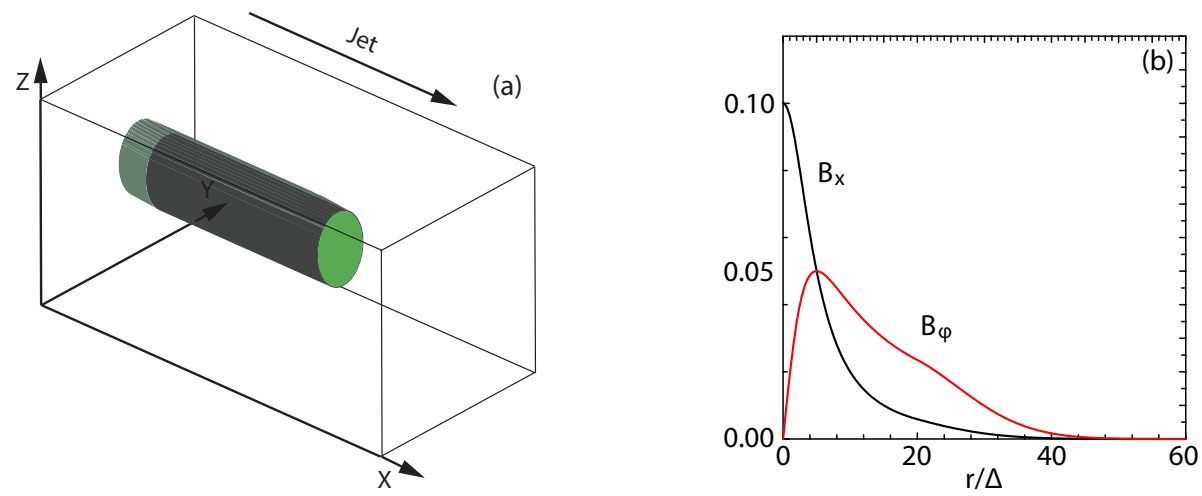

Figure 1. Panel (a) shows the schematic global jet simulation setup used in this simulation study. The jet is injected at $x=100 \Delta$ with jet radius $r_{\mathrm{jt}}=20 \Delta$ at the center of the $y-z$ plane (not scaled; adapted from [14]); Panel (b) shows the magnetic field component profiles across the jet. The field structure is defined by Equations (1) and (2), with damping applied outside of the jet with length-scale $b=200.0$ (see text). The jet boundary is located at $r_{\text {jet }}=20 \Delta$. The size of the simulation box is small in these simulations, which are meant to investigate an initial stage of the system evolution. 
In these small system simulations, we utilize a numerical grid with $\left(L_{x}, L_{y}, L_{z}\right)=(645 \Delta, 131 \Delta, 131 \Delta)$ (simulation cell size: $\Delta=1$ ) and periodic boundary conditions in transverse directions. The jet and ambient (electron) plasma number density measured in the simulation frame is $n_{\mathrm{jt}}=8$ and $n_{\mathrm{am}}=12$, respectively. The cylindrical jet with jet radius $r_{j \mathrm{j}}=20 \Delta$ is injected in the middle of the $y-z$ plane $\left(\left(y_{\mathrm{jc}}, z_{\mathrm{jc}}\right)=(63 \Delta, 63 \Delta)\right)$ at $x=100 \Delta$.

In the simulations, initial magnetic field amplitude parameter $B_{0}=0.1 c,(c=1)$, $\left(\sigma=B^{2} / n_{\mathrm{e}} m_{\mathrm{e}} \gamma_{\mathrm{jet}} c^{2}=2.8 \times 10^{-3}\right)$, and $a=5.0 \Delta=0.25 * r_{\mathrm{jt}}\left(r_{\mathrm{jt}}=20 \Delta\right)$. The helical field structure inside the jet is defined by Equations (1) and (2). For the external magnetic fields, we use a damping function $\exp \left[-\left(r-r_{\mathrm{jt}}\right)^{2} / b\right]\left(r \geq r_{\mathrm{jt}}\right)$ that multiplies Equations (1) and (2) with the tapering parameter $b=200$. The final profile of the helical magnetic field components is shown in Figure $1 b$.

In the simulations, the electron skin depth $\lambda_{\mathrm{s}}=c / \omega_{\mathrm{pe}}=10.0 \Delta$, where $c$ is the speed of light, $\omega_{\mathrm{pe}}=\left(e^{2} n_{\mathrm{am}} / \epsilon_{0} m_{\mathrm{e}}\right)^{1 / 2}$ is the electron plasma frequency, and the electron Debye length for the ambient electrons is $\lambda_{\mathrm{D}}=0.5 \Delta$. The jet-electron thermal velocity is $v_{\mathrm{jt}, \mathrm{th}, \mathrm{e}}=0.014 \mathrm{c}$ in the jet reference frame. The electron thermal velocity in the ambient plasma is $v_{\mathrm{am}, \mathrm{th}, \mathrm{e}}=0.03 c$, and ion thermal velocities are smaller by $\left(m_{\mathrm{i}} / m_{\mathrm{e}}\right)^{1 / 2}$. Simulations were performed using an electron-positron $\left(e^{ \pm}\right)$plasma or an electron-proton $\left(e^{-}-p^{+}\right.$with $\left.m_{\mathrm{p}} / m_{\mathrm{e}}=1836\right)$ plasma for the jet Lorentz factor of 15 and with the ambient plasma at rest $\left(v_{\mathrm{am}}=0\right)$.

Figure 2 shows isocontour plots of the $x$-component of the current density $J_{\mathrm{x}}$ for the (a) $e^{-}-p^{+}$and (b) $e^{ \pm}$jets at time $t=500 \omega_{\mathrm{pe}}^{-1}(y / \Delta=63)$. For the $e^{-}-p^{+}$jet, recollimation-like shocks can be observed (Figure 2a).

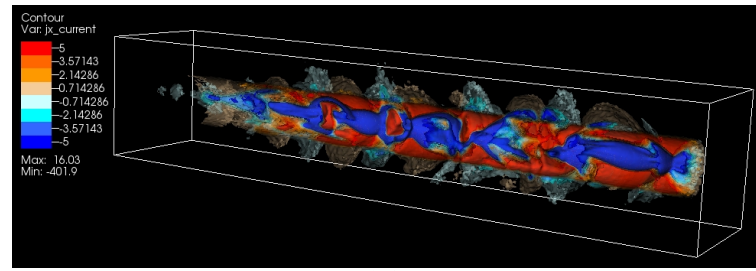

(a)

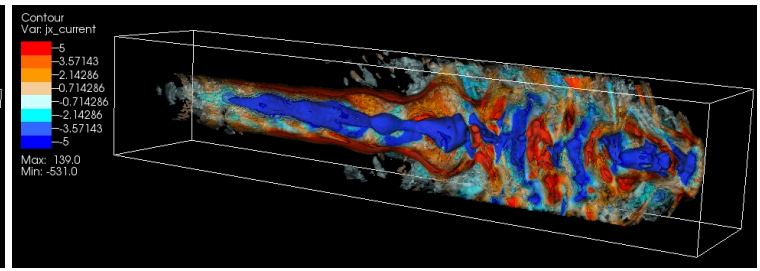

(b)

Figure 2. Isocontour plots of $J_{\mathrm{X}}$ for $(\mathbf{a}) e^{-}-p^{+}$; and (b) $e^{ \pm}$jets at time $t=500 \omega_{\mathrm{pe}}^{-1}$. For the $e^{-}-p^{+}$jet, recollimation-like shocks are seen in Figure $2 a$. Figure $2 b$ shows the growing instabilities and currents expanding outside the jet, leading to a turbulent current density structure for the $e^{ \pm}$jet.

The negative current density (blue) is disrupted by the positive current density (red), which indicates the occurrence of recollimations, like in RMHD simulations [33]. In contrast, for the $e^{ \pm}$jet, small recollimation structures occur, and after instabilities have grown, the currents expand outside the jet and the current density becomes turbulent.

Figure 3 shows the magnetic field lines (white lines) which are carried by the jets. The helical magnetic fields are distorted due to the instabilities occurring at the jet boundaries (see Figure 2).

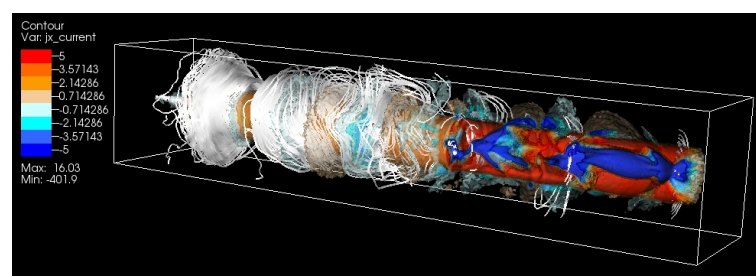

(a)

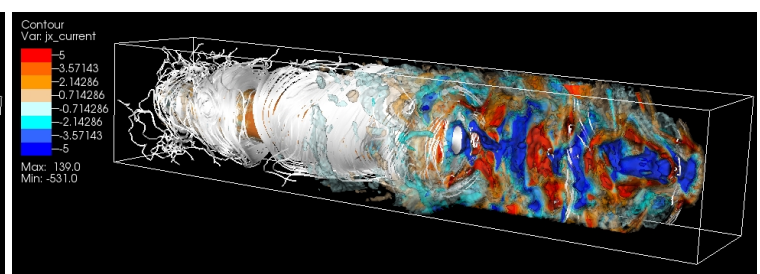

(b)

Figure 3. Isocontour plots of $J_{\mathrm{x}}$ with magnetic field lines (white lines) for (a) $e^{-}-p^{+}$; and (b) $e^{ \pm}$jets at time $t=500 \omega_{\mathrm{pe}}^{-1}$. In order to view the inside of the jets, 3D displays are clipped along the jets and perpendicular to the jets in the front parts of the figures (compare Figure 2). 
Figure 4 shows the $y$ component of the magnetic field $\left(B_{y}\right)$. In both cases, the initial helical magnetic field (left-handed; clockwise viewed from the jet front) is enhanced and disrupted due to the instabilities.

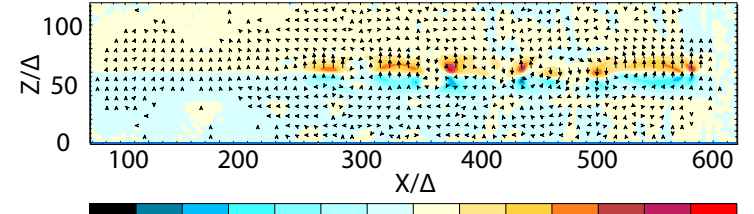

(a)

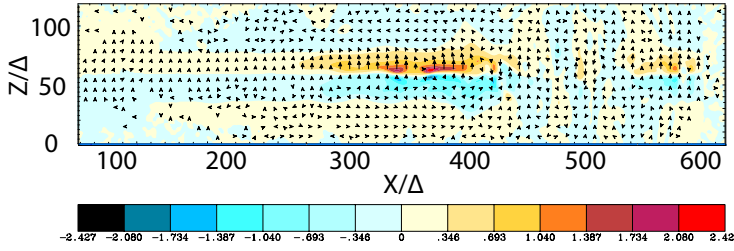

(b)

Figure 4. Isocontour plots of the azimuthal component of magnetic field $B_{y}$ intensity at the center of the jets for for $(\mathbf{a}) e^{-}-p^{+}$and $(\mathbf{b}) e^{ \pm}$jets $(y / \Delta=63)$ at time $t=500 \omega_{\mathrm{pe}}^{-1}$. The disruption of helical magnetic fields is caused by instabilities and/or reconnection.

\subsection{Particle Acceleration in Helically Magnetized RPIC Jets}

In order to examine particle acceleration, we analyze jet electron phase-space distributions in $x-\gamma v_{x}, \gamma v_{y}$. Figure 5 shows phase-space plots for (a) $e^{-}-p^{+}$and (b) $e^{ \pm}$jets at time $t=500 \omega_{\text {pe }}^{-1}$. For comparison purposes, the figure includes phase-space plots for simulations without magnetic fields (panels (c) and (d)). The red dots show $x-\gamma v_{x}$, and the blue dots show $x-\gamma v_{y}$ phase-space distributions. The phase-space distributions indicate strong electron acceleration and deceleration. In the case of the $e^{-}-p^{+}$jet (Figure 5a), the acceleration and deceleration occurs both along and transverse to the jet direction. This result is reminiscent of recollimation shocks observed in RMHD simulations [33].

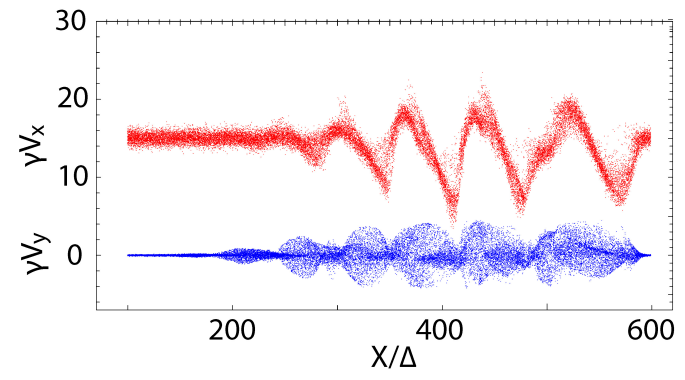

(a)

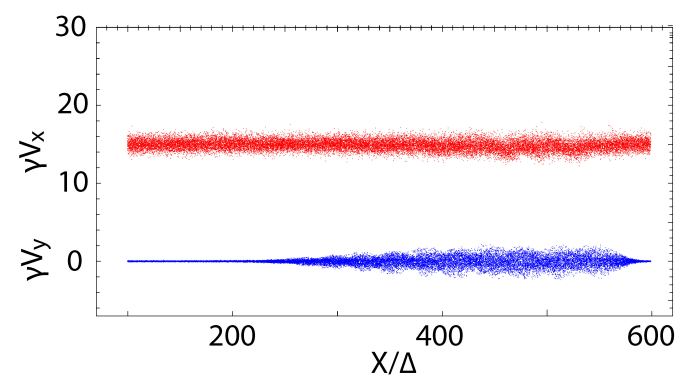

(c)

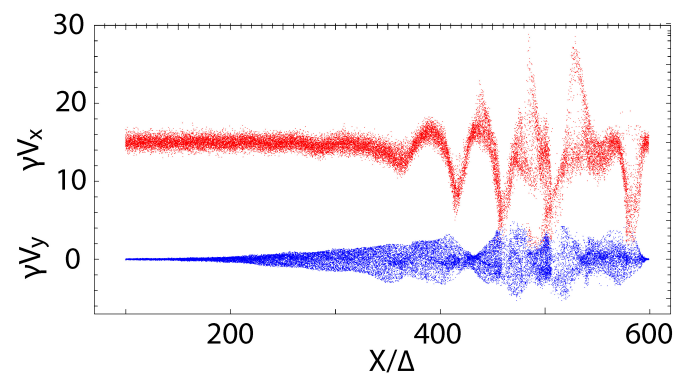

(b)

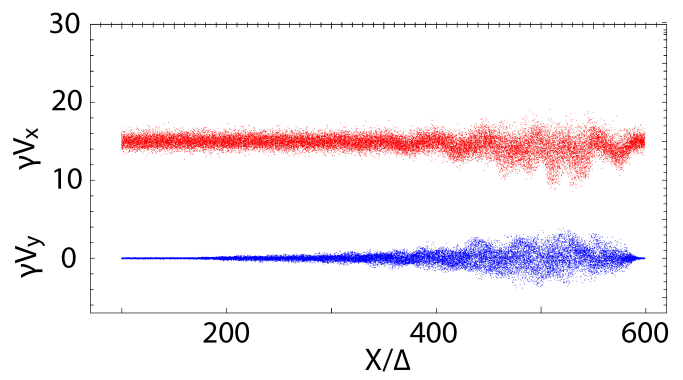

(d)

Figure 5. Phase-space plots of jet electrons (red dots: $x-\gamma v_{x}$, and blue dots: $x-\gamma v_{y}$ ) for (a) $e^{-}-p^{+}$; and (b) $e^{ \pm}$jets at time $t=500 \omega_{\mathrm{pe}}^{-1}$. Panels (c) and (d) show phase-space plots for simulations without helical magnetic fields. The strong velocity variation in longitudinal and transverse directions can be caused by recollimation shocks and/or other new instabilities. 
On the contrary, in the case of the $e^{ \pm}$jet, the phase-space structure is more complicated and suggests that reconnection and/or some new instabilities occur. Further investigation using larger simulation systems is clearly needed to resolve these issues.

\section{Comparison to RMHD Results for Jets Containing Helical Magnetic Fields}

In order to compare our RPIC simulation results with RMHD simulations of (a) recollimation and (b) current-driven kink instability, two typical RMHD cases are plotted in Figure 6. Figure 6a shows the enhanced Lorentz factor due to recollimation shocks found by Mizuno et al. [33] in two-dimensional special-relativistic magnetohydrodynamic simulations of non-equilibrium over-pressured helically-magnetized relativistic jets in cylindrical geometry. Similar structures are observed in our simulations (Figures $2 a$ and $4 a$ ). Figure $6 c$ shows the Lorentz factor of jet electrons with the observed enhanced Lorentz factor due to the recollimation shocks. As expected from Figure 5a, the location of the enhanced Lorentz factor corresponds to the location of the jet electrons, which are accelerated maximally in longitudinal and transverse directions. However, the evolution of the enhanced Lorentz factor is different compared to the RMHD simulation result. The movie of a 2D slice of $J_{x}$ like Figure 2a shows that the recollimation shocks are moving along the jet, keeping the shape.
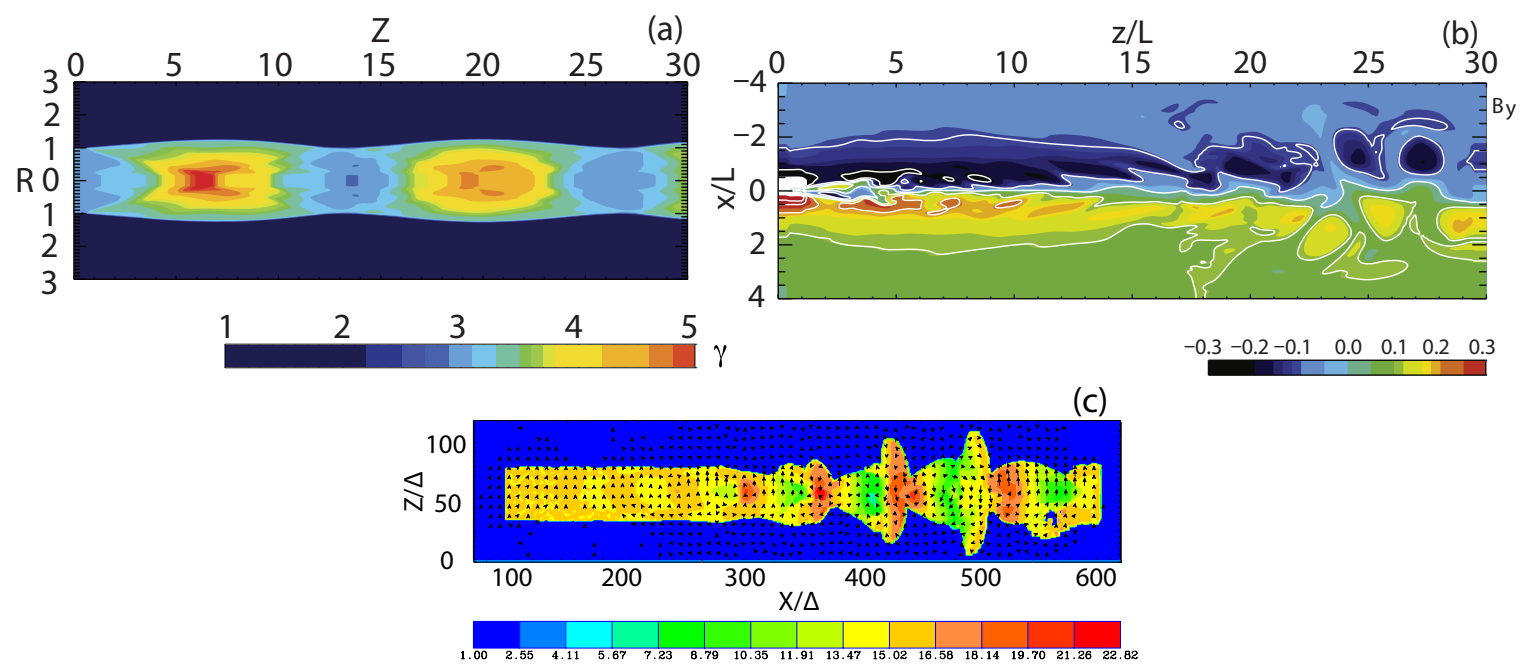

Figure 6. Panel (a) shows a 2D plot of the Lorentz factor for a helically-magnetized RMHD jet at $t=200 r_{\mathrm{jt}} / c$ (adapted from Figure 10d in Mizuno et al. [33] ); Panel (b) shows the azimuthal magnetic field component $B_{y}$ with $\left|B_{y}\right|$ magnitude contours for a decreasing density helically-magnetized rotating RMHD jet at $t=70 r_{\mathrm{jt}} / c$. The disruption of helical magnetic fields can be caused by the current-driven kink instability (adapted from Figure $4 \mathrm{f}$ in Singh et al. [31]); Panel (c) shows the Lorentz factor of jet electrons for $e^{-}-p^{+}$jet $(y / \Delta=63)$ at time $t=500 \omega_{\mathrm{pe}}^{-1}$.

Recently, Singh et al. [31] showed the spatial development of the current-driven kink instability along magnetized rotating relativistic jets. Figure $6 \mathrm{~b}$ shows the azimuthal magnetic field component $B_{y}$ with $\left|B_{y}\right|$ magnitude contours with right-hand polarity of helical magnetic field (counter-clockwise, viewed from the jet front). Therefore, the direction of $B_{y}$ is reversed. The current-driven kink instability breaks simple helical magnetic fields. This structure in $B_{y}$ is similar to that of the $e^{ \pm}$jet case, as shown in Figure $4 b$.

\section{Discussion}

Our initial global jet simulations containing helical magnetic fields show new types of growing instabilities for both electron-proton and pair plasma jets. Preliminary results indicate that the presence of helical fields suppresses the growth of the kinetic instabilities, such as the Weibel instability, kKHI, 
and MI. Instead, new instabilities appear, associated with recollimation shocks and current-driven kink instability.

The $e^{-}-p^{+}$helically magnetized jet shows recollimation-like shock structures in the current density $J_{x}$, similar to recollimation shocks observed in RMHD simulations containing helical magnetic fields [33]. The observed modulations in the kinetic energy of jet electrons shown in Figure 5a might correspond to the modulations in the Lorentz factor reported in RMHD studies (see Figure 6a). Additionally, while not shown here, the electron density in the $e^{-}-p^{+}$jet shows pile-ups which correspond to recollimation shock structures seen in RMHD simulations. Evidence for the growth of a kink-like instability in the $e^{ \pm}$jet is seen in the $y$-component of magnetic field $B_{y}$ in Figure $4 \mathrm{~b}$, and is similar to that seen in Figure $6 b$, where helical magnetic fields carried by the jet are disrupted by the growth of the kink instability.

Finally, we see evidence that reconnection is taking place in the jets. However, larger-scale and higher-resolution simulations are required to fully resolve the reconnection phenomena and to understand the nature of the new instabilities. Future simulations will be combined with calculations of radiation signatures and polarity, along with variations in space and time $[34,35]$.

Supplementary Materials: The following are available online at http://www.mdpi.com/2075-4434/4/4/38/s1, Video S1: Evolution of $J_{x}$ for the $e^{-}-p^{+}$jet.

Acknowledgments: This work is supported by NSF AST-0908010, AST-0908040, NASA-NNX09AD16G, NNX12AH06G, NNX13AP-21G, and NNX13AP14G grants. The work of J.N. and O.K. has been supported by Narodowe Centrum Nauki through research project DEC-2013/10/E/ST9/00662. Y.M. is supported by the ERC Synergy Grant “BlackHoleCam-Imaging the Event Horizon of Black Holes” (Grant No. 610058). M.P. acknowledges support through grant PO 1508/1-2 of the Deutsche Forschungsgemeinschaft. Simulations were performed using Pleiades and Endeavor facilities at NASA Advanced Supercomputing (NAS), and using Gordon and Comet at The San Diego Supercomputer Center (SDSC), and Stampede at The Texas Advanced Computing Center, which are supported by the NSF. This research was started during the program "Chirps, Mergers and Explosions: The Final Moments of Coalescing Compact Binaries" at the Kavli Institute for Theoretical Physics, which is supported by the National Science Foundation under grant No. PHY05-51164. The first velocity shear results using an electron-positron plasma were obtained during the Summer Aspen workshop "Astrophysical Mechanisms of Particle Acceleration and Escape from the Accelerators" held at the Aspen Center for Physics (1-15 September 2013).

Author Contributions: Ken-Ichi Nishikawa: Perform simulations, analyze the data and prepare a manuscript; Yosuke Mizuno: Compare with RMHD simulations; Jacek Niemiec: Contribute modifying the code for this research; Oleh Kobzar; Modify the code for this simulation; Martin Pohl: Overlook the simulation results; Jose L. Gómez: Contribute on comparisons with observations; Ioana Duţan: Perform some of simulations for this research; Asaf Pe'er: Critical contributions for physical interpretation; Jacob Trier Frederiksen: Contribution for critical discussions on this research; Åke Nordlund: Fruitful suggestions for this research; Athina Meli: Critical reading and discussion on this research; Helene Sol: Essential suggestions for this research; Philip E. Hardee: Theoretical contributions for this research; Dieter. H. Hartmann: Useful discussions for this research.

Conflicts of Interest: The authors declare no conflict of interest.

\section{References}

1. Pe'er, A. Energetic and Broad Band Spectral Distribution of Emission from Astronomical Jets. Space Sci. Rev. 2014, 183, 371-403.

2. Blandford, R.D.; Znajek, R.L. Electromagnetic extraction of energy from Kerr black holes. Mon. Not. R. Astron. Soc. 1977, 179, 433-456.

3. McKinney, J.C.; Tchekhovskoy, A.; Sadowski, A.; Narayan, N. Three-dimensional general relativistic radiation magnetohydrodynamical simulation of super-Eddington accretion, using a new code HARMRAD with M1 closure. Mon. Not. R. Astron. Soc. 2014, 441, 3177-3208.

4. Laing, R.A. Magnetic fields in extragalactic radio sources. Astrophys. J. 1981, 248, 87-104.

5. Aloy, M.; Gómez, J.; Ibáñez, J.M.; Martí, J.M.; Müller, E. Radio Emission from Three-dimensional Relativistic Hydrodynamic Jets: Observational Evidence of Jet Stratification. Astrophys. J. 2000, 528, L85-L88.

6. Clausen-Brown, E.; Lyutikov, M.; Kharb, P. Signatures of large-scale magnetic fields in active galactic nuclei jets: Transverse asymmetries. Mon. Not. R. Astron. Soc. 2011, 415, 2081-2092.

7. Meier, D.L. Astrophysics: Exhaust inspection. Nature 2008, 452, 945-946. 
8. Piran, T. The physics of gamma-ray bursts. Rev. Mod. Phys. 2004, 76, 1143-1214.

9. O'Sullivan, S.P.; McClure-Griffiths, N.M.; Feain, I.J.; Gaensler, B.M.; Saul, R.J. Broad-band radio circular polarization spectrum of the relativistic jet in PKS B2126-158. Mon. Not. R. Astron. Soc. 2013, 435, 311-319.

10. Wardle, J.F.C.; Homan, D.C.; Ojha, R.; Roberts, D.H. Electron-positron jets associated with the quasar 3C279. Nature 1998, 395, 457-461.

11. Buneman, O. Computer Space Plasma Physics: Simulation Techniques and Software; Matsumoto, H., Omura, Y., Eds.; Terra Scientific Publishing Company: Tokyo, Japan, 1993; pp. 67-79.

12. Niemiec, J.; Pohl, M.; Stroman, T.; Nishikawa, K.-I. Production of Magnetic Turbulence by Cosmic Rays Drifting Upstream of Supernova Remnant Shocks. Astrophys. J. 2008, 684, 1174-1189.

13. Nishikawa, K.-I.; Niemiec, J.; Medvedev, M.; Sol, H.; Hardee, P.; Mizuno, Y.; Zhang, B.; Pohl, M.; Oka, M.; Hartmann, D.H. Weibel instability and associated strong fields in a fully 3D simulation of a relativistic shock. Astrophys. J. Lett. 2009, 698, L10-L14.

14. Nishikawa, K.-I.; Frederiksen, J.T.; Nordlund, Å.; Mizuno, Y.; Hardee, P.E.; Niemiec, J.; Gómez, J.L.; Pe'er, A.; Duţan, I.; Meli, A.; et al. Evolution of Global Relativistic Jets: Collimations and Expansion with kKHI and the Weibel Instability. Astrophys. J. 2016, 820, 94-107.

15. Alves, E.P.; Grismayer, T.; Martins, S.F.; Fiúza, F.; Fonseca, R.A.; Silva, L.O. Large-scale magnetic field generation via the Kelvin-Helmholtz instability in unmagnetized scenarios. Astrophys. J. Lett. 2012, 746, L14-L19.

16. Alves, E.P.; Grismayer, T.; Fonseca, R.A.; Silva, L.O. Electron-scale shear instabilities: Magnetic field generation and particle acceleration in astrophysical jets. New J. Phys. 2014, 16, 035007.

17. Alves, E.P.; Grismayer, T.; Fonseca, R.A.; Silva, L.O. Transverse electron-scale instability in relativistic shear flows. Phys. Rev. E 2015, 92, 021101.

18. Grismayer, T.; Alves, E.P.; Fonseca, R.A.; Silva, L.O. dc-Magnetic-Field Generation in Unmagnetized Shear Flows. Phys. Rev. Lett. 2013, 111, 015005.

19. Grismayer, T.; Alves, E.P.; Fonseca, R.A. Silva, L.O. Theory of multidimensional electron-scale instabilities in unmagnetized shear flows. Plasma Phys. Control. Fusion 2013, 55, 124031.

20. Nishikawa, K.-I.; Hardee, P.; Mizuno, Y.; Dutan, I.; Zhang, B.; Medvedev, M.; Choi, E.J.; Min, K.W.; Niemiec, J.; Nordlund, A.; et al. Radiation from relativistic jets from particles accelerated by shocks, shear-flows, and reconnection. In Proceedings of the EPJ Web of Conferences, Granada, Spain, 10-14 June 2013; Volume 61, 02003.

21. Nishikawa, K.-I.; Zhang, B.; Dutan, I.; Medvedev, M.; Hardee, P.; Choi, E.-J.; Min, K.; Niemiec, J.; Mizuno, Y.; Nordlund, A.; et al. Magnetic field generation in a jet-sheath plasma via the kinetic Kelvin-Helmholtz instability. Ann. Geophys. 2013, 31, 1535-1541.

22. Nishikawa, K.-I.; Hardee, P.; Dutan, I.; Niemiec, J.; Medvedev, M.; Mizuno, Y.; Meli, A.; Sol, H.; Zhang, B.; Pohl, M.; et al. Magnetic field generation via the kinetic Kelvin-Helmholtz instability in core-sheath jets. Astrophys. J. 2014, 793, 163-248.

23. Liang, E.; Böttcher, M.; Smith, I. Magnetic Field Generation and Particle Energization at Relativistic Shear Boundaries in Collisionless Electron-Positron Plasmas. Astrophys. J. Lett. 2013, 766, L19-L24.

24. Liang, E.; Fu, W.; Böttcher, M.; Smith, I.; Roustazadeh, P. Relativistic Positron-Electron-Ion Shear Flows and Application to Gamma-Ray Bursts. Astrophys. J. Lett. 2013, 779, L27-L34.

25. Alves, E.P. Magnetic Field Generation via the Kelvin-Helmholtz Instability. Master's Thesis, Universidate Témica de Lisbon, Lisboa, Portugal, 2010.

26. Nishikawa, K.-I.; Hardee, P.; Dutan, I.; Zhang, B.; Meli, A.; Chjoi, E.-J.; Min, K.; Niemiec, J.; Mizuno, Y.; Medvedev, M.; et al. Radiation from Particles Accelerated in Relativistic Jet Shocks and Shear-fows. In Proceedings of the 2014 Fifth International Fermi Symposium eConf C14102.1, Nagoya, Japan, 20-24 October 2014.

27. Nishikawa, K.-I.; Hardee, P.; Richardson, G.; Preece, R.; Sol, H.; Fishman, G.J. Particle Acceleration in Relativistic Jets Due to Weibel Instability. Astrophys. J. 2003, 595, 555-563.

28. Nishikawa, K.-I.; Hardee, P.; Richardson, G.; Preece, R.; Sol, H.; Fishman, G.J. Particle Acceleration and Magnetic Field Generation in Electron-Positron Relativistic Shocks. Astrophys. J. 2005, 622, 927-937.

29. Ng, J.S.T.; Noble, R.J. Inductive and Electrostatic Acceleration in Relativistic Jet-Plasma Interactions. Phys. Rev. Lett. 2006, 96, 115006.

30. Mizuno, Y.; Hardee, P.E.; Nishikawa, K.-I. Spatial Growth of the Current-Driven Instability in Relativistic Jets. Astrophys. J. 2014, 784, 167. 
31. Singh, C.B.; Mizuno, Y.; de Gouveia Dal Pino, E.M. Spatial Growth of Current-driven Instability in Relativistic Rotating Jets and the Search for Magnetic Reconnection. Astrophys. J. 2016, 824, 48.

32. Markidis, S.; Lapenta, G.; Delzanno, G.L.; Henri, P.; Goldman, M.V.; Newman, D.L.; Intrator, T.; Laure, E. Signatures of secondary collisionless magnetic reconnection driven by kink instability of a flux rope. Plasma Phys. Control. Fusion 2014, 56, 064010.

33. Mizuno, Y.; Gómez, J.L.; Nishikawa, K.-I.; Meli, A.; Hardee, P.E.; Rezzolla, L. Recollimation Shocks in Magnetized Relativistic Jets. Astrophys. J. 2015, 809, 38.

34. Zhang, H.; Chen, X.; Böttcher, M.; Guo, F.; Li, H. Polarization Swings Reveal Magnetic Energy Dissipation in Blazars. Astrophys. J. 2015, 804, 60-68.

35. Zhang, H.; Deng, W.; Li, H.; Böttcher, M. Polarization Signatures of Relativistic Magnetohydrodynamic Shocks in the Blazar Emission Region. I. Force-free Helical Magnetic Fields. Astrophys. J. 2016, 817, 63.

(C) 2016 by the authors; licensee MDPI, Basel, Switzerland. This article is an open access article distributed under the terms and conditions of the Creative Commons Attribution (CC-BY) license (http://creativecommons.org/licenses/by/4.0/). 